OPEN ACCESS

Edited by:

Laszlo Csiba

University of Debrecen, Hungary

Reviewed by:

Viktor Berczi,

Semmelweis University, Hungary

Odysseas Kargiotis,

Metropolitan Hospital, Greece

*Correspondence:

Yingqi Xing

xingyq2009@sina.com

Li Cui

Icui@jlu.edu.cn

Specialty section:

This article was submitted to

Applied Neuroimaging,

a section of the journal

Frontiers in Neurology

Received: 28 October 2021 Accepted: 24 January 2022

Published: 17 February 2022

Citation:

Zhang J, Wang L, Chen Y, Wang S, Xing $Y$ and Cui L (2022) Color Doppler Ultrasonography for the Evaluation of Subclavian Artery Stenosis. Front. Neurol. 13:804039. doi: 10.3389/fneur.2022.804039

\section{Color Doppler Ultrasonography for the Evaluation of Subclavian Artery Stenosis}

\author{
Jie Zhang ${ }^{1}$, Lijuan Wang ${ }^{1}$, Ying Chen ${ }^{1}$, Sibo Wang ${ }^{1}$, Yingqi Xing ${ }^{2 *}$ and Li Cui ${ }^{1 *}$ \\ ${ }^{1}$ Neuroscience Center, Department of Neurology, The First Hospital of Jilin University, Jilin University, Changchun, China, \\ ${ }^{2}$ Department of Vascular Ultrasonography, Xuanwu Hospital, Capital Medical University, Beijing, China
}

Background: It is of great significance to evaluate symptomatic subclavian artery (SA) stenosis by color Doppler ultrasonography. More than 50\% SA stenosis may induce symptoms. Currently, there is a paucity of published literature and lack of practitioner consensus for how ultrasonic findings should be interpreted in patients with SA stenosis.

Objective: The study aimed to prospectively evaluate SA stenosis using color Doppler ultrasonography, with digital subtraction angiography as a reference. Moreover, we aimed to determine the optimal thresholds to predict SA stenosis ( $\geq 50 \%)$.

Methods: A total of 423 SAs from 234 patients with normal or stenotic lumen were enrolled. The peak systolic velocity (PSV) and acceleration time at the stenotic and distal segments of the SA, peak reversed velocity of the vertebral artery, and waveforms of the stenotic SA, distal SA, and vertebral artery were recorded. The ratios of stenotic PSV to distal PSV (PSVr) and distal AT to stenotic AT were also calculated. The optimal cutoff values were determined using receiver operating characteristic analysis.

Results: All ultrasonic parameters were significantly correlated with the degree of SA stenosis, whereas PSV $(r=0.624, P<0.001)$, PSVr $(r=0.654, P<0.001)$ and VA waveform change $(r=0.631, P<0.001)$ had the strongest correlation with SA stenosis. The optimal cutoff values were as follows: PSV $\geq 230 \mathrm{~cm} / \mathrm{s}$ and $\mathrm{PSV} r \geq 2.2$ to predict $\geq$ $50 \%$ stenosis, and PSV $\geq 340 \mathrm{~cm} / \mathrm{s}$ and PSVr $\geq 3.0$ to predict $\geq 70 \%$ stenosis.

Conclusions: Symptomatic patients with the ultrasonic parameters of PSV $\geq 230 \mathrm{~cm} / \mathrm{s}$ and PSVr $\geq 2.2$ need to be considered for further verification by computed tomographic angiography or magnetic resonance angiography, or by digital subtraction angiography with a view to percutaneous transluminal angioplasty/stent implantation in the same session. The recommended graded cutoff values can help in long-term management of patients with SA stenosis.

Keywords: color Doppler ultrasonography, subclavian artery stenosis, subclavian steal syndrome, vertebrobasilar insufficiency, digital subtraction angiography 


\section{INTRODUCTION}

The prevalence of subclavian artery (SA) disease is $\sim 2 \%$ in the general population, $42 \%$ in patients with documented peripheral artery disease, and $5 \%$ in patients referred for coronary artery bypass grafting (CABG). In patients with SA disease, half have concomitant coronary artery disease and one-third have carotid and/or vertebral artery (VA) disease (1-3). SA stenosis is a marker of atherosclerotic disease and increased risk of cardiovascular, cerebrovascular, and peripheral vascular events (3-8). In symptomatic patients with SA stenosis, surgical or interventional treatment should be considered $(9,10)$. Indications include upper extremity claudication, vertebrobasilar insufficiency, symptoms of myocardial ischemia in patients who underwent $\mathrm{CABG}$ utilizing the internal mammary artery, and lower extremity claudication in patients who underwent axillo-femoral bypass. Treatment should also be considered to increase blood flow before surgical procedures, such as CABG or the creation of dialysis arteriovenous fistula $(1,11,12)$. Thus, accurate evaluation of SA stenosis is of great importance, especially symptomatic SA stenosis.

There are several non-invasive methods for evaluating SA stenosis. Measuring the blood pressure in both arms is a convenient and effective method of assessing SA stenosis. A systolic cuff pressure difference of $>10 \mathrm{mmHg}$ is considered significant. However, there is a high false positive rate in patients with hypertension (13). And the accuracy and precision of blood pressure measurement varies across different measurement sites (14). Radial pulse palpation is another useful clinical examination. But it depends on changes in blood pressure and its accuracy is questionable $(15,16)$. The current guidelines rate color Doppler ultrasonography (CDU) as appropriate for the evaluation of a patient with suspected subclavian occlusive disease (17). Ultrasonic assessment of stenotic SA enables the detection of high-velocity flow and monophasic post-stenotic flow in SA, and reversed flow in ipsilateral VA (10). To date, there is a lack of practitioner consensus for ultrasonic evaluation of symptomatic SA stenosis and how ultrasonic findings should be interpreted in patients with SA stenosis. Two previous studies only evaluated $\geq 70 \%$ stenosis of SA by CDU $(18,19)$. However, $\geq 70 \%$ stenosis of $\mathrm{SA}$ is not equivalent to symptomatic stenosis. Even 50-69\% SA stenosis can induce symptoms, especially when myocardial or upper extremity flow demand increases $(4,20)$. It is necessary to establish ultrasonic criteria to predict symptomatic SA stenosis.

Therefore, the aim of this study was to prospectively evaluate SA stenosis using CDU, with digital subtraction angiography (DSA) as the reference. We aimed to validate the diagnostic efficacy of CDU and assess the optimal thresholds for $\geq 50 \%$ SA stenosis.

\section{MATERIALS AND METHODS}

\section{Population}

From April 2020 to August 2021, 239 consecutive patients with symptomatic SA stenosis or carotid artery stenosis who were scheduled to undergo DSA at our institution were prospectively enrolled (Figure 1). All the patients had undergone computed tomographic angiography (CTA) or magnetic resonance angiography (MRA) examination, which confirmed subclavian artery or carotid artery stenosis. The subsequent DSA was performed with a view to endovascular percutaneous transluminal angioplasty (PTA) or stent implantation in the same session. All patients were fully informed of the procedure and provided informed consent. They were evaluated by blinded examiners, first by CDU, and then by DSA, within an interval of $24 \mathrm{~h}$. Data on patient demographics and vascular risk factors were collected after CDU. Symptomatic subclavian stenosis was defined as upper extremity claudication, vertebrobasilar insufficiency, myocardial ischemia in patients who underwent coronary artery bypass grafting utilizing the internal mammary artery, and lower extremity claudication in patients who underwent axillo-femoral bypass. The exclusion criteria were as follows: (i) SA occlusion, (ii) previous SA surgery or stenting, and (iii) atrial fibrillation or other arrhythmias affecting velocity assessment. The study protocol was approved by the ethics committee of the First Hospital of Jilin University on March 20, 2020 (approval number: 2020-632). The study was completed in compliance with the current privacy regulations.

\section{Ultrasonic Examination}

For CDU, an iU22 system (Philips Healthcare, Bothell, WA) with a 9-3 $\mathrm{MHz}$ linear array probe and 5-1 $\mathrm{MHz}$ curvilinear array probe was used. According to the ultrasound protocol previously described by Pellerito (21), all CDUs were performed by the same physician (Z. J.), who had 8 years of experience in vascular ultrasonography, had performed more than 2000 vascular cases per year, and who was unaware of the patient's clinical data (other imaging data, physical examination results, laboratory results, and patient history). The ultrasound parameters measured were peak systolic velocity (PSV) and acceleration time (AT) at the stenotic segment of the SA, peak systolic velocity (PSVd) and acceleration time (ATd) at the distal segment of the SA, and peak reversed velocity (PRV) of the VA. The spectral waveforms of the stenotic SA, distal SA, and VA were recorded. The ratios of PSV to PSVd (PSVr) and ATd to AT (ATr) were also calculated. AT represents the time from systolic acceleration to peak flow. PRV represents the value from the baseline to the reversed systolic peak of VA. The waveforms of the stenotic and distal SA included the triphasic, biphasic, and monophasic waveforms. The waveforms of the VA included the unchanged, mid-systolic notch, bidirectional, and completely retrograde waveforms (Figure 2).

\section{DSA Examination}

DSA was performed using AXIOM Artis dBa (Siemens, Erlangen, Germany). All catheterizations were performed using a transfemoral approach with standard diagnostic catheters. After aortic arch injection, selective supra-aortic (carotid and subclavian) artery injections were performed. Angiography was interpreted by two experienced vascular physicians (C. Y. and W. S.), who had at least 5 years of experience in endovascular therapy. If the results were inconsistent, the final decision was made by another senior physician (W. L.), who had 12 years of 


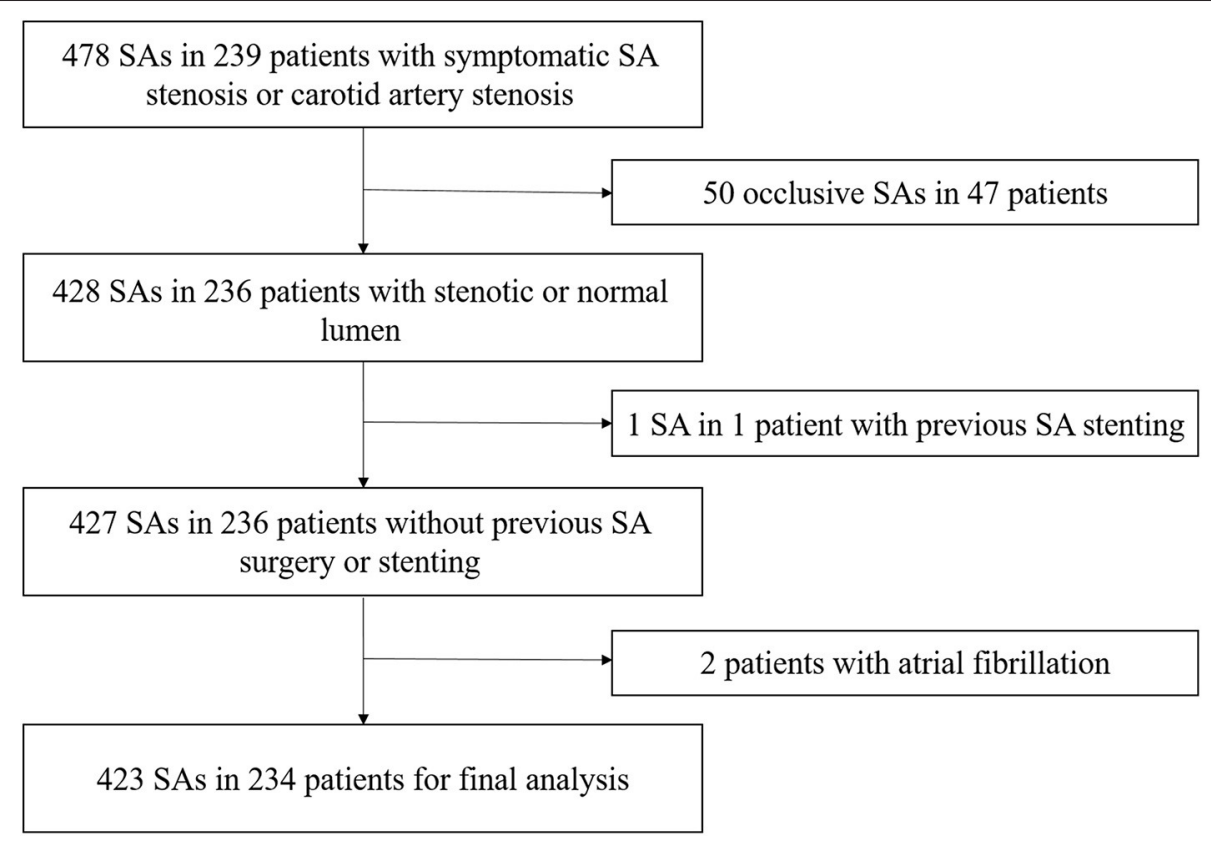

FIGURE 1 | Study inclusion/exclusion criteria. SA, subclavian artery.
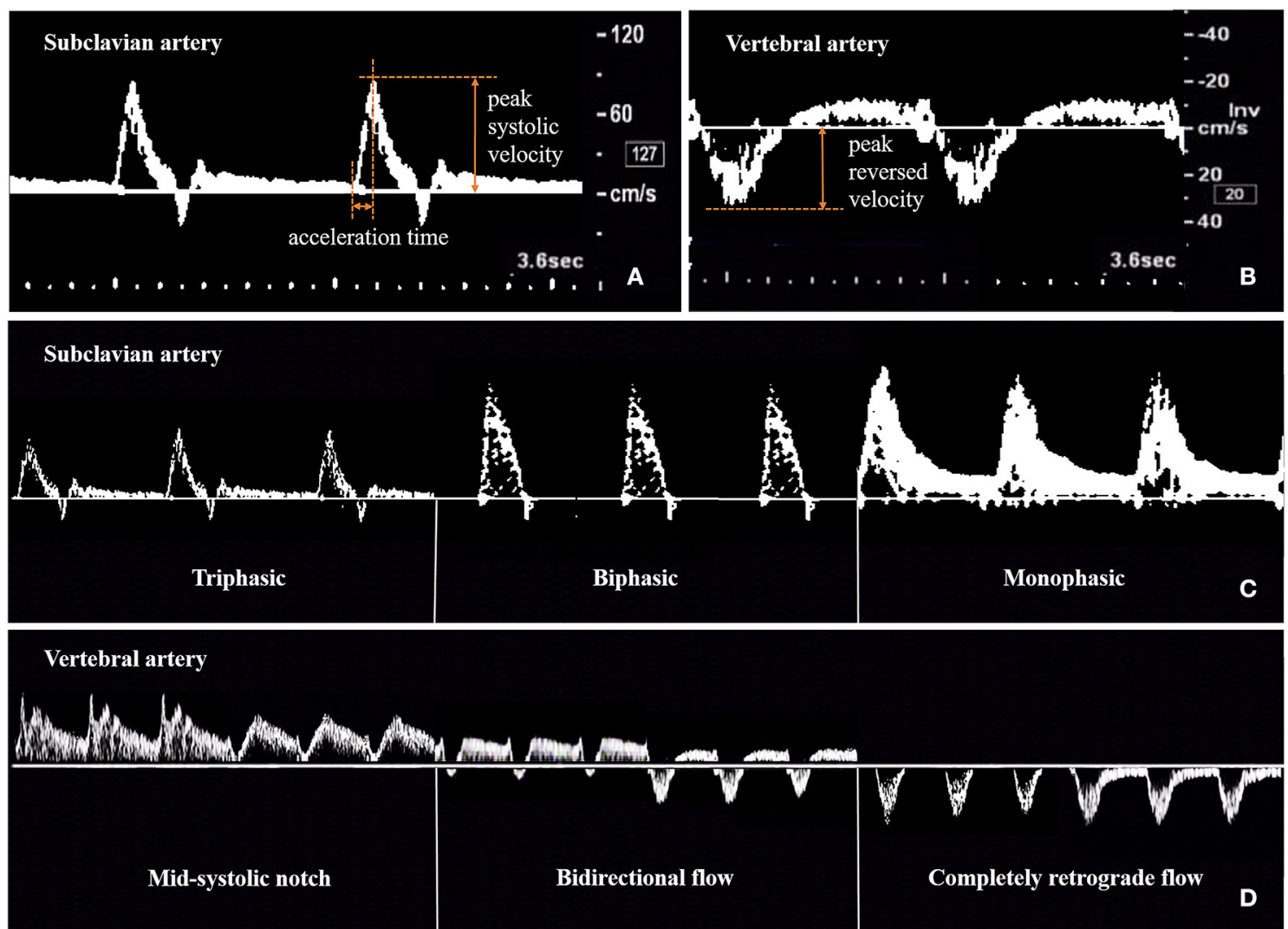

FIGURE 2 | Method for measuring and recording ultrasonic parameters of subclavian artery (A,C) and vertebral artery (B,D) 
TABLE 1 | Demographic characteristics.

\begin{tabular}{|c|c|c|c|c|c|}
\hline \multirow[b]{2}{*}{ Variable } & \multicolumn{3}{|c|}{ Degree of stenosis } & \multirow[b]{2}{*}{$\mathrm{F} / \chi^{2} / \mathrm{H}$ value } & \multirow[b]{2}{*}{$P$-value } \\
\hline & Normal $(n=325)$ & $50-69 \%(n=50)$ & $70-99 \%(n=48)$ & & \\
\hline Age (years) & $62.8 \pm 9.3$ & $62.9 \pm 8.1$ & $63.5 \pm 7.5$ & 0.124 & 0.883 \\
\hline Male & 266 (81.8\%) & 38 (76.0\%) & 34 (70.8\%) & 3.698 & 0.157 \\
\hline \multicolumn{6}{|l|}{ Comorbidity } \\
\hline Hypertension & $210(64.6 \%)$ & $30(60.0 \%)$ & 23 (47.9\%) & 5.073 & 0.079 \\
\hline Diabetes mellitus & 102 (31.4\%) & $16(32.0 \%)$ & 12 (25.0\%) & 0.844 & 0.656 \\
\hline Dyslipidemia & 102 (31.4\%) & $20(40.0 \%)$ & 17 (35.4\%) & 1.618 & 0.445 \\
\hline Coronary artery disease & 49 (15.1\%) & $5(10.0 \%)$ & 7 (14.6\%) & 0.906 & 0.636 \\
\hline Smoking & 185 (56.9\%) & 28 (56.0\%) & 23 (47.9\%) & 1.376 & 0.502 \\
\hline Symptomatic & 0 (0.00\%) & $25(50.0 \%)^{\star}$ & $29(60.4 \%)^{\star}$ & 207.675 & $<0.001$ \\
\hline
\end{tabular}

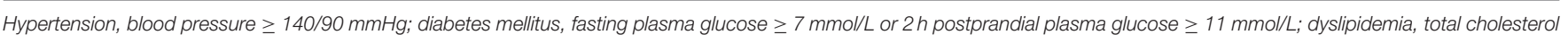

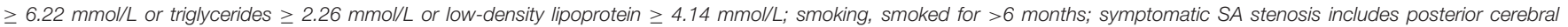
insufficiency and arm ischemia. ${ }^{\star}$ Compared with the normal group, $P<0.05$.

endovascular therapy experience. All physicians were blinded to the ultrasound examination findings and other imaging data. The degree of SA stenosis was recorded, which was determined using the North American Symptomatic Carotid Endarterectomy Trial criteria (22). The degree of SA stenosis was subdivided into three categories: normal, 50-69\%, and 70-99\%.

\section{Statistical Analysis}

Normality of distribution was assessed using the KolmogorovSmirnov test. Continuous variables are expressed as mean \pm standard deviation or median (interquartile range), and categorical variables are expressed as frequencies and percentages. Categorical variables were tested using the $\chi^{2}$ or Fisher's exact tests, and continuous variables were tested using one-way analysis of variance or the Kruskal-Wallis test. The Spearman's rank correlation coefficient was used to calculate the relationship between the degree of SA stenosis and hemodynamic parameters. The predictive values (sensitivity and specificity) were calculated. The optimal thresholds of various hemodynamic parameters for SA stenosis were determined based on the maximum Youden's index by receiver operating characteristic curve analysis. Statistical comparisons of the area under the receiver operating characteristic curve (AUC) of the different hemodynamic parameters were performed according to Delong's test (23). Statistical significance was set at $P<0.05$. The SPSS (version 26.0, IBM Corp, Armonk, NY) and MedCalc (version 19.5.6 for Windows, Mariakerke, Belgium) software were used to conduct the statistical analyses.

\section{RESULTS}

\section{Baseline Data}

A total of 478 SAs in 239 patients were enrolled, including 78 patients with symptomatic SA stenosis and 161 patients with symptomatic carotid stenosis. Among the 156 SAs in 78 patients with symptomatic SA stenosis, 91 stenotic arteries and 65 contralateral normal arteries were included. Forty-three (55.1\%) patients had concomitant carotid artery disease. Among the
322 SAs in 161 patients with symptomatic carotid stenosis, 57 asymptomatic stenotic SAs and 265 normal arteries were included. Overall, these patients included 148 stenotic SAs and 330 normal SAs. According to the exclusion criteria in Figure 1, a total of 98 stenotic SAs and 325 normal SAs were enrolled finally. No complications of DSA occurred. With combined use of the linear and curvilinear array probes, all the ultrasound parameters of enrolled patients were obtained. According to the results of the DSA, 50 (11.8\%) had 50-69\% stenosis, 48 (11.3\%) had 7099\% stenosis, and 325 (76.8\%) had normal lumen. Among the stenotic SAs, Eighty-nine (90.8\%) SA stenosis were located at the origin, six $(6.1 \%)$ were located near the origin of the VA, two $(2.0 \%)$ were located distal to the VA, and one $(1.0 \%)$ were multiple stenosis. Fifty-five (56.1\%) SA stenosis coexisted with ipsilateral VA stenosis, two (2.0\%) with ipsilateral VA hypoplasia, 28 (28.6\%) with contralateral VA stenosis, 29 (29.6\%) with contralateral SA stenosis, and one $(1.0 \%)$ with an ipsilateral VA originating from the aortic arch. Fifty-four (55.1\%) stenotic SAs were symptomatic, including 50 SAs with posterior cerebral insufficiency, two SAs with arm ischemia, and two SAs with coexistence of posterior cerebral insufficiency and arm ischemia. The proportion of symptomatic SA stenosis was the highest in the $70-99 \%$ stenosis group (60.4\%), but this was not significantly different $(P=0.318)$ from the proportion in the $50-69 \%$ stenosis group $(50.0 \%)$. Demographic characteristics are summarized in Table 1. There were no significant differences in the baseline data between the different groups $(P>0.05)$.

\section{Hemodynamic Parameters}

The features of SA stenosis detected by CDU were increased PSV, spectral broadening, and extended AT in the SA Doppler, a change from a triphasic waveform to a biphasic or monophasic waveform at the stenotic SA and distal segment, and mid-systolic notch or reverse flow at the VA. The hemodynamic characteristics are summarized in Table 2. A correlation was observed between the degree of SA stenosis and PSV $(r=0.624, P<0.001)$, PSVd $(r=-0.313, P<0.001)$, PSVr $(r=0.654, P<0.001)$, AT $(r=0.171, P<0.001)$, ATd $(r=0.255, P<0.001)$, and 
TABLE 2 | Hemodynamic characteristics.

\begin{tabular}{|c|c|c|c|c|c|}
\hline \multirow[b]{2}{*}{ Variable } & \multicolumn{3}{|c|}{ Degree of stenosis } & \multirow[b]{2}{*}{$\mathrm{F} / \chi^{2} / \mathrm{H}$ value } & \multirow[b]{2}{*}{$P$-value } \\
\hline & Normal $(n=325)$ & $50-69 \%(n=50)$ & $70-99 \%(n=48)$ & & \\
\hline PSV (cm/s) & $147.0(102.5)$ & $350.5(165.8)^{\star}$ & $419.5(196.8)^{\star}$ & 164.233 & $<0.001$ \\
\hline PSVr & $1.3(0.6)$ & $3.0(2.5)^{\star}$ & $6.2(4.1)^{*}{ }^{\dagger}$ & 180.983 & $<0.001$ \\
\hline AT (ms) & $50.0(20.0)$ & $50.0(40.0)$ & $70.0(50.0)^{*}{ }^{*}$ & 18.904 & $<0.001$ \\
\hline ATd (ms) & $50.0(20.0)$ & $50.0(32.5)$ & $105(77.5)^{\star}{ }^{\dagger}$ & 46.436 & $<0.001$ \\
\hline ATr & $1.0(0.5)$ & $1.0(0.5)$ & $1.4(0.8)^{\star}{ }^{\dagger}$ & 13.937 & 0.001 \\
\hline \multicolumn{6}{|c|}{ Waveform of stenotic subclavian artery } \\
\hline Triphasic & $284(87.4 \%)$ & $24(48.0 \%)^{\star}$ & $10(20.8 \%)^{*}$ & 121.707 & $<0.001$ \\
\hline Biphasic & $41(12.6 \%)$ & $15(30.0 \%)^{\star}$ & $4(8.3 \%)^{\dagger}$ & 12.282 & 0.003 \\
\hline Monophasic & $0(0.0 \%)$ & $11(22.0 \%)^{\star}$ & $34(70.8 \%)^{*}$ & 228.433 & $<0.001$ \\
\hline \multicolumn{6}{|c|}{ Waveform of the distal subclavian artery } \\
\hline Triphasic & $186(57.2 \%)$ & $14(28.0 \%)^{\star}$ & $5(10.4 \%)^{*}$ & 46.204 & $<0.001$ \\
\hline Biphasic & 139 (42.8\%) & $28(56.0 \%)$ & $11(22.9 \%)^{\star}$ & 11.271 & 0.003 \\
\hline Monophasic & $0(0.0 \%)$ & $8(16.0 \%)^{\star}$ & $32(66.7 \%)^{*}$, & 155.970 & $<0.001$ \\
\hline \multicolumn{6}{|c|}{ Waveform of vertebral artery } \\
\hline Unchanged & $323(99.4 \%)$ & $38(76.0 \%)^{\star}$ & $17(35.4 \%)^{*}$ & 190.667 & $<0.001$ \\
\hline Mid-systolic notch & $2(0.6 \%)$ & $9(18.0 \%)^{\star}$ & $11(22.9 \%)^{\star}$ & 50.147 & $<0.001$ \\
\hline Bidirectional & $0(0.0 \%)$ & $3(6.0 \%)^{*}$ & $10(20.8 \%)^{\star}$ & 42.618 & $<0.001$ \\
\hline Completely retrograde & $0(0.0 \%)$ & 0 (0.0\%) & $10(20.8 \%)^{*}$ & 41.664 & $<0.001$ \\
\hline
\end{tabular}

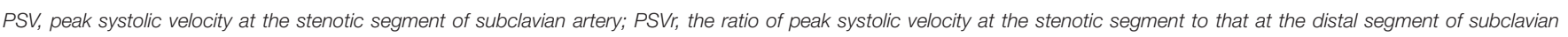

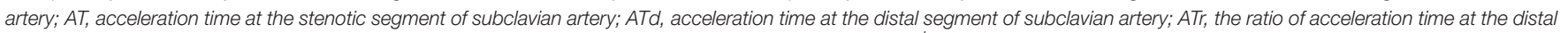
segment to that at the stenotic segment of subclavian artery. ${ }^{\star}$ Compared with the normal group, $P<0.05$. ${ }^{\dagger}$ Compared with the $50-69 \%$ stenosis group, $P<0.05$.

$\operatorname{ATr}(r=0.128, P=0.009)$. The waveforms (triphasic, biphasic, and monophasic) at the stenotic SA $(r=-0.587, P<0.001)$ and distal SA $(r=-0.470, P<0.001)$ were also related to the degree of SA stenosis. Forty-three SA stenosis $(43 / 98,43.9 \%)$ had concomitant ipsilateral VA spectrum changes. A correlation was found between the degree of SA stenosis and waveform of VA $(r$ $=0.631, P<0.001)$ and PRV $(r=0.483, P<0.001)$. The presence of ipsilateral VA spectrum changes was significantly correlated with symptomatic subclavian stenosis $(P<0.001)$.

\section{Cutoff Values}

The receiver operating characteristic curves of SA flow for evaluating SA stenosis are shown in Figure 3. All AUCs were $>0.90$. For $\geq 50 \%$ stenosis, there was no significant difference between the AUCs of PSV and PSVr $(P=0.0872)$. The cutoff values were PSV $\geq 230 \mathrm{~cm} / \mathrm{s}$ (sensitivity of 88.8 and specificity of $81.2 \%$ ) and PSVr $\geq 2.2$ (sensitivity of $86.7 \%$ and specificity of $86.5 \%)$. The sensitivity and specificity of changes of SA spectrum for predicting $\geq 50 \%$ stenosis were 65.3 and $87.4 \%$, respectively. The sensitivity and specificity of changes of VA spectrum for predicting $\geq 50 \%$ stenosis were 43.9 and $99.4 \%$, respectively. The parameters of SA flow were more sensitive than changes of SA or VA spectrum (all $P<0.001$ ), but the change of VA spectrum was the most specific parameter (all $P<0.001$ ). For $\geq 70 \%$ stenosis, the AUC of PSVr was larger than that of PSV $(P=$ 0.0012 ). The cutoff values were PSV $\geq 340 \mathrm{~cm} / \mathrm{s}$ (sensitivity of $79.2 \%$ and specificity of $90.7 \%$ ) and PSVr $\geq 3.0$ (sensitivity of $93.8 \%$ and specificity of $89.3 \%$ ). The sensitivity and specificity of changes of SA spectrum for predicting $\geq 70 \%$ stenosis were 79.2 and $82.1 \%$, respectively. The sensitivity and specificity of changes of VA spectrum for predicting $\geq 70 \%$ stenosis were 64.6 and $96.3 \%$, respectively. The parameter of PSVr was the most sensitive parameter (all $P<0.05$ ), and the parameter of change of VA spectrum was the most specific parameter (all $P<0.01$ ).

\section{DISCUSSION}

In this study, we prospectively enrolled symptomatic patients with SA stenosis or carotid artery stenosis, and analyzed various ultrasonic parameters to predict SA stenosis. The results showed that CDU is an effective non-invasive method for evaluating SA stenosis. Furthermore, we established the grading ultrasonic diagnostic criteria for SA stenosis, with DSA as the reference.

\section{Ultrasound Parameters in Previous Studies}

$\mathrm{CDU}$ is an appropriate evaluation method for patients with suspected subclavian occlusive disease. The ultrasound parameters for predicting SA stenosis include direct and indirect parameters. Most previous studies have focused on indirect parameters, including PRV and waveform changes of stenotic SA, distal SA, and VA. In the studies on changes of ipsilateral VA waveform, SA stenosis was predicted by evaluating retrograde VA flow (20, 24-28). Retrograde VA flow does not occur in all cases of SA stenosis. In our study, $43.9 \%$ of the SA stenosis had concomitant ipsilateral VA spectrum changes. Although the VA spectrum changes were related to 

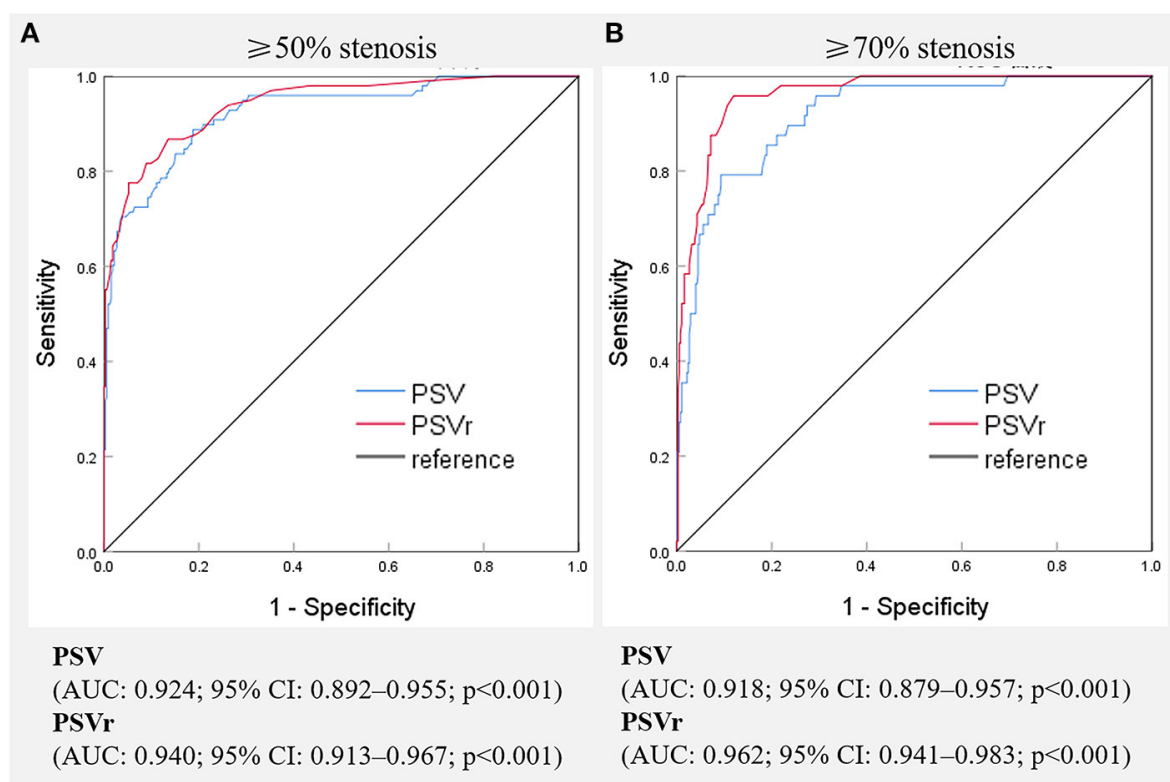

FIGURE 3 | Receiver operating characteristic curves of various hemodynamic parameters for the evaluation of $\geq 50 \%(\mathbf{A})$ and $\geq 70 \%(\mathbf{B})$ stenosis of the subclavian artery. PSV, peak systolic velocity; PSVr, the ratio of peak systolic velocity at the stenotic segment to that at the distal segment; AUC, area under receiver operating characteristic curve, $\mathrm{Cl}$, confidence interval.

the degree of SA stenosis $(r=0.631, P<0.001)$, only $24.0 \%$ of the moderate SA stenosis and $64.6 \%$ of the severe SA stenosis were combined with VA spectrum changes. This was partly due to the high incidence of blocked VA-VA stealing pathway. The causes included ipsilateral VA stenosis (56.1\%), contralateral VA stenosis $(28.6 \%)$, contralateral SA stenosis (29.6\%), SA stenosis located near or distal to the VA (8.2\%), ipsilateral VA hypoplasia (2.0\%), and ipsilateral VA originating from the aortic arch (1.0\%). Although all the enrolled SAs were from patients who were scheduled to undergo DSA, which might cause selection bias, our study showed that it was not sufficiently sensitive to use the spectral change of VA to predict SA stenosis in patients who required further consideration for interventional therapy. In addition, changes in the VA spectrum do not always indicate a subclavian steal phenomenon $(29,30)$. The damped and monophasic changes at distal waveforms in the upper extremity are also a sign of significant SA stenosis, but this finding may be present in healthy patients with low-resistance arm circulation (31). In the current study, waveform changes of distal SA correlated with SA stenosis, but the correlation was not as strong as other parameters. Two previous studies evaluated the direct parameters to predict exceeding 70\% stenosis of SA, including PSV and PSVr $(18,19)$. Their studies showed conflicting results. Hua et al. (19) evaluated 252 SAs and recommended a PSV of $\geq 343 \mathrm{~cm} / \mathrm{s}$ and PSVr of $\geq 4.0$ for evaluating $\geq 70 \%$ stenosis, while Mousa et al. (18) evaluated 245 SAs and found that a PSV of $>240$ $\mathrm{cm} / \mathrm{s}$ had good sensitivity to detect $>70 \%$ stenosis. The cutoff values of PSV differed greatly, which would worry the clinicians about the practical application of ultrasound parameters. More importantly, surgical or interventional treatment is indicated for symptomatic patients with $\geq 50 \%$ SA stenosis. Thus, the evaluation of $\geq 50 \%$ stenosis is more meaningful, but there is no relevant literature report. In addition, several studies found that AT was an useful ultrasound parameter to predict internal carotid artery stenosis, lower extremity peripheral artery stenosis, renal artery stenosis, hepatic artery stenosis and coronary artery stenosis (32-36). However, its efficacy in evaluating SA stenosis is unclear. In the current study, we found that the parameters of AT, ATd, and ATr were significantly correlated with SA stenosis, but the correlation was not as strong as other parameters.

\section{Ultrasound Parameters in Current Study}

We evaluated the correlation between SA stenosis and all ultrasound parameters, including direct and indirect parameters. We found that all parameters were significantly correlated with the degree of SA stenosis, with PSV $(r=0.624, P<0.001)$, PSVr $(r=0.654, P<0.001)$, change of SA waveform $(r=$ $0.587, P<0.001)$, and change of VA waveform $(r=0.631$, $P<0.001)$ having the strongest correlation. Furthermore, we determined the cutoff values of PSV and PSVr for detecting $\geq 50 \%$ and $\geq 70 \%$ SA stenosis, and calculated the sensitivity and specificity of parameters of VA and SA waveform changes. Our focus was about the cutoff value of $\geq 50 \%$ stenosis. The parameters of PSV and PSVr were the most sensitive parameters, and change of VA spectrum was the most specific parameter (all $P<0.001$ ). Based on the maximum Youden's index, PSV and PSVr were more appropriate parameters to predict $\geq 50 \%$ SA stenosis. In addition, owing to the conflicting results of the two previous studies on $\geq 70 \%$ SA stenosis, we conducted the prospective study with a relatively large sample size to verify 
these results. In the present study, we examined 423 SAs and recommended a PSV of $\geq 340 \mathrm{~cm} / \mathrm{s}$ and PSVr of $\geq 3.0$ to detect $>70 \%$ stenosis. PSVr was the most sensitive parameter (all $P$ $<0.05$ ), and change of VA spectrum was the most specific parameter (all $P<0.01$ ). Based on the maximum Youden's index, PSVr was the most appropriate parameter to predict $\geq 70 \%$ SA stenosis.

\section{Clinical Significance}

Revascularization is recommended in patients with symptomatic SA stenosis, so the identification of symptomatic SA stenosis is of great importance. As shown in Table 1, patients with $\geq 50 \%$ SA stenosis may be symptomatic, and there was no statistical difference in the proportion of symptomatic patients in the 50$69 \%$ and $70-99 \%$ SA stenosis groups. In our study, PSV and PSVr were more appropriate parameters to predict $\geq 50 \%$ SA stenosis. The cutoff values were PSV $\geq 230 \mathrm{~cm} / \mathrm{s}$ and PSVr $\geq 2.2$. Therefore, if the patient has symptoms such as upper extremity claudication, vertebrobasilar insufficiency, myocardial ischemia with CABG, and lower extremity claudication with axillo-femoral bypass, and the ultrasonic flow parameters of PSV $\geq 230 \mathrm{~cm} / \mathrm{s}$ and PSVr $\geq 2.2$, they should be considered for further verification by CTA or MRA, or by DSA with a view to $\mathrm{PTA} /$ stent implantation in the same session. In addition, even asymptomatic SA stenosis is associated with an increased risk of morbidity and mortality related to underlying atherosclerotic disease burden in other vascular beds (4-7). These patients should be treated aggressively with antiplatelet agents, highdose statins, and antihypertensive agents (1). Consequently, SA stenosis, whether symptomatic or asymptomatic, mild or severe, should be closely monitored the progress of the lesion. In our study, we added hemodynamic assessment of $\geq 70 \%$ SA stenosis, which would be helpful for the clinical long-term management of patients with SA stenosis.

\section{Limitations}

\section{Our Study Has Several Limitations}

First, hyperemia-ischemia cuff test was not performed in this study, which was a useful screening tool for the detection of subclavian stenosis causing VA steal syndrom. Due to the high prevalence of VA stenosis in patients enrolled in the study, we did not evaluate its utility in the current study. We will evaluate the efficacy of hyperemia-ischemia cuff test in evaluating SA stenosis in the further. Second, the patients with SA occlusion were excluded, which were part of patients with symptomatic SA disease. We excluded the patients with SA occlusion, since no flow seen on CDU at the occlusive segment of subclavian artery and several direct ultrasound parameters could not be detected. In the future, we will carry out the study of comprehensive

\section{REFERENCES}

1. Saha T, Naqvi SY, Ayah OA, McCormick D, Goldberg S. Subclavian artery disease: diagnosis and therapy. Am J Med. (2017) 130:409-16. doi: 10.1016/j.amjmed.2016.12.027

2. Abdul Jabbar A, Houston J, Burket M, Il'Giovine ZJ, Srivastava BK, Agarwal A. Screening for subclinical subclavian artery stenosis before coronary ultrasound evaluation of SA occlusion to explore the clinical significance of ultrasound parameters of occlusive SA. Finally, all enrolled patients in this study were from the stroke unit of the Department of Neurology at the First Hospital of Jilin University. As this study was conducted in a single department at a single center, it may have caused selection bias. The patients in this study were mainly those with vertebrobasilar insufficiency and upper extremity claudication, while those with myocardial ischemia after CABG were not included. In the future, we will conduct a multi-center and multi-departmental prospective study to verify these results.

In conclusion, our study found that CDU is a reliable imaging modality for evaluating SA stenosis. Symptomatic patients with the ultrasonic parameters of PSV $\geq 230 \mathrm{~cm} / \mathrm{s}$ and $\mathrm{PSVr} \geq$ 2.2 need to be considered for further verification by CTA or MRA, or by DSA with a view to PTA/stent implantation in the same session.

\section{DSA to Seek Revascularization}

The recommended graded cutoff values can help in long-term management of patients with SA stenosis.

\section{DATA AVAILABILITY STATEMENT}

The raw data supporting the conclusions of this article will be made available by the authors, without undue reservation.

\section{ETHICS STATEMENT}

The studies involving human participants were reviewed and approved by Ethics Committee of The First Hospital of Jilin University. The patients/participants provided their written informed consent to participate in this study.

\section{AUTHOR CONTRIBUTIONS}

JZ contributed to the study conception and design, data collection, analysis and interpretation, and drafting of the manuscript. YX and LC contributed to the study conception and design, analysis and interpretation of the data, and revision of the manuscript. SW, YC, and LW contributed to the data collection and revision of the manuscript. All authors contributed to the article and approved the submitted version.

\section{ACKNOWLEDGMENTS}

The authors acknowledge all the patients for their participation in this study.

artery bypass grafting: should we do it? Echocardiography. (2017) 34:928-33. doi: $10.1111 /$ echo. 13528

3. Shadman R, Criqui $\mathrm{MH}$, Bundens WP, Fronek A, Denenberg JO, Gamst AC, et al. Subclavian artery stenosis: prevalence, risk factors, and association with cardiovascular diseases. $J$ Am Coll Cardiol. (2004) 44:618-23. doi: 10.1016/j.jacc.2004. 04.044 
4. Potter BJ, Pinto DS. Subclavian steal syndrome. Circulation. (2014) 129:23203. doi: 10.1161/CIRCULATIONAHA.113.006653

5. Labropoulos N, Nandivada P, Bekelis K. Prevalence and impact of the subclavian steal syndrome. Ann Surg. (2010) 252:166-70. doi: 10.1097/SLA.0b013e3181e3375a

6. Aboyans V, Kamineni A, Allison MA, McDermott MM, Crouse JR, Ni H, et al. The epidemiology of subclavian stenosis and its association with markers of subclinical atherosclerosis: the multi-ethnic study of atherosclerosis (MESA). Atherosclerosis. (2010) 211:266-70. doi: 10.1016/j.atherosclerosis.2010.01.013

7. Clark CE, Taylor RS, Shore AC, Ukoumunne OC, Campbell JL. Association of a difference in systolic blood pressure between arms with vascular disease and mortality: a systematic review and meta-analysis. Lancet. (2012) 379:905-14. doi: 10.1016/S0140-6736(11)61710-8

8. Ochoa VM, Yeghiazarians Y. Subclavian artery stenosis: a review for the vascular medicine practitioner. Vasc Med. (2011) 16:29-34. doi: 10.1177/1358863X10384174

9. Brott TG, Halperin JL, Abbara S, Bacharach JM, Barr JD, Bush RL, et al. 2011 ASA/ACCF/AHA/AANN/AANS/ACR/ASNR/CNS/SAIP/SCAI/SIR/SNIS/ SVM/SVS guideline on the management of patients with extracranial carotid and vertebral artery disease: a report of the American college of cardiology foundation/American heart association task force on practice guidelines, and the American stroke association, american association of neuroscience nurses, American association of neurological surgeons, American college of radiology, American society of neuroradiology, congress of neurological surgeons, society of atherosclerosis imaging and prevention, society for cardiovascular angiography and interventions, society of interventional radiology, society of neurointerventional surgery, society for vascular medicine, and society for vascular surgery. J Am Coll Cardiol. (2011) 124:489-532. doi: 10.1161/CIR.0b013e31820d8d78

10. Aboyans V, Ricco JB, Bartelink MEL, Bjorck M, Brodmann M, Cohnert T, et al. 2017 ESC guidelines on the diagnosis and treatment of peripheral arterial diseases, in collaboration with the European society for vascular surgery (ESVS): document covering atherosclerotic disease of extracranial carotid and vertebral, mesenteric, renal, upper and lower extremity arteriesendorsed by: the European stroke organization (eso)the task force for the diagnosis and treatment of peripheral arterial diseases of the European society of cardiology (ESC) and of the European society for vascular surgery (ESVS). Eur Heart J. (2018) 39:763-816. doi: 10.1093/eurheartj/ehx095

11. Varotto L, De Boni A, Iannucci G, Caprioglio F. Uncommon intravascular lithotripsy for the treatment of subclavian steal syndrome: a case presenting with concomitant acute coronary syndrome and transient ischaemic attack. Eur Heart J. (2021). doi: 10.1093/eurheartj/ehaa1082

12. Moccetti F, Brinkert M, Wolfrum M, Toggweiler S. Coronary subclavian steal syndrome. Eur Heart J. (2020) 41:1345. doi: 10.1093/eurheartj/ehz832

13. Yu Y, Liu L, Lo K, Tang S, Feng Y. Prevalence and associated factors of inter-arm blood pressure difference in Chinese community hypertensive population. Postgrad Med. (2021) 133:188-94. doi: 10.1080/00325481.2020.1826184

14. Siaron KB, Cortes MX, Stutzman SE, Venkatachalam A, Ahmed KM, Olson DM. Blood Pressure measurements are site dependent in a cohort of patients with neurological illness. Sci Rep. (2020) 10:3382. doi: 10.1038/s41598-020-60414-7

15. Schmidt WA, Gromnica-Ihle E. What is the best approach to diagnosing large-vessel vasculitis? Best Pract Res Clin Rheumatol. (2005) 19:223-42. doi: 10.1016/j.berh.2005.01.006

16. Sabharwal A, Strickland T, Yentis SM. Ability of radial arterial palpation and observation of the pulse oximetry trace to estimate non-invasive systolic pressure in healthy volunteers and in women undergoing spinal anaesthesia for elective caesarean section. Anaesthesia. (2011) 66:20-4. doi: 10.1111/j.1365-2044.2010.06586.x

17. American College of Cardiology F, American College of R, American Institute of Ultrasound in M, American Society of E, American Society of $\mathrm{N}$, Intersocietal Commission for the Accreditation of Vascular L, et al. ACCF/ACR/AIUM/ASE/ASN/ICAVL/SCAI/SCCT/SIR/SVM/SVS/SVU [corrected] 2012 appropriate use criteria for peripheral vascular ultrasound and physiological testing part I: arterial ultrasound and physiological testing: a report of the American college of cardiology foundation appropriate use criteria task force, American college of radiology, American institute of ultrasound in medicine, American society of echocardiography, American society of nephrology, intersocietal commission for the accreditation of vascular laboratories, society for cardiovascular angiography and interventions, society of cardiovascular computed tomography, society for interventional radiology, society for vascular medicine, society for vascular surgery, [corrected] and society for vascular ultrasound. [corrected]. J Am Coll Cardiol. (2012) 60:242-76. doi: 10.1016/j.jacc.2012.02.009

18. Mousa AY, Morkous R, Broce M, Yacoub M, Sticco A, Viradia R, et al. Validation of subclavian duplex velocity criteria to grade severity of subclavian artery stenosis. J Vasc Surg. (2017) 65:1779-85. doi: 10.1016/j.jvs.2016.12.098

19. Hua Y, Jia L, Li L, Ling C, Miao Z, Jiao L. Evaluation of severe subclavian artery stenosis by color doppler flow imaging. Ultrasound Med Biol. (2011) 37:358-63. doi: 10.1016/j.ultrasmedbio.2010.12.003

20. Suma S, Coli S, Palumbo A, Graziuso S, Borrello B, Gaibazzi N. Vertebral doppler bunny waveform suggesting subclavian stenosis and influencing coronary artery bypass technique. Tex Heart Inst J. (2020) 47:70-1. doi: 10.14503/THIJ-17-6406

21. Pellerito J. Introduction to Vascular Ultrasonography 6th Edition. Philadelphia, PA: Saunders (2012). p. 260-80.

22. North American Symptomatic Carotid Endarterectomy Trial C, Barnett HJM, Taylor DW, Haynes RB, Sackett DL, Peerless SJ, et al. Beneficial effect of carotid endarterectomy in symptomatic patients with high-grade carotid stenosis. N Engl J Med. (1991) 325:445-53. doi: 10.1056/NEJM199108153250701

23. DeLong ER, DeLong DM, Clarke-Pearson DL. Comparing the areas under two or more correlated receiver operating characteristic curves: a nonparametric approach. Biometrics. (1988) 44:837-45. doi: 10.2307/2531595

24. Policha A, Baldwin M, Lee V, Adelman MA, Rockman C, Berland T, et al. Clinical significance of reversal of flow in the vertebral artery identified on cerebrovascular duplex ultrasound. J Vasc Surg. (2018) 67:568-72. doi: 10.1016/j.jvs.2017.07.113

25. Chen SP, Hu YP. Waveform patterns and peak reversed velocity in vertebral arteries predict severe subclavian artery stenosis and occlusion. Ultrasound Med Biol. (2015) 41:1328-33. doi: 10.1016/j.ultrasmedbio.2014.12.019

26. Sakima H, Wakugawa Y, Isa K, Yasaka M, Ogata T, Saitoh M, et al. Correlation between the degree of left subclavian artery stenosis and the left vertebral artery waveform by pulse doppler ultrasonography. Cerebrovasc Dis. (2010) 31:64-7. doi: 10.1159/000320853

27. Kliewer MA, Hertzberg BS, Kim DH, Bowie JD, Courneya DL, Carroll BA. Vertebral artery Doppler waveform changes indicating subclavian steal physiology. AJR Am J Roentgenol. (2000) 174:815-9. doi: 10.2214/ajr.174.3.1740815

28. Paivansalo M, Heikkila O, Tikkakoski T, Leinonen S, Merikanto J, Suramo I. Duplex ultrasound in the subclavian steal syndrome. Acta Radiol. (1998) 39:183-8. doi: 10.1080/02841859809172176

29. Chen SP, Hu YP, Fan LH, Guan LJ. Completely reversed flow in the vertebral artery does not always indicate subclavian steal phenomenon. Ultrasound Med Biol. (2014) 40:1072-82. doi: 10.1016/j.ultrasmedbio.2013. 12.004

30. Chen S-P, Hu Y-P, Fan L-H, Zhu X-L. Bidirectional flow in the vertebral artery is not always indicative of the subclavian steal phenomenon. J Ultrasound Med. (2013) 32:1945-50. doi: 10.7863/ultra.32.11.1945

31. Kalaria VG, Jacob S, Irwin W, Schainfeld RM. Duplex ultrasonography of vertebral and subclavian arteries. J Am Soc Echocardiogr. (2005) 18:1107-11. doi: 10.1016/j.echo.2005.06.005

32. Strosberg DS, Haurani MJ, Satiani B, Go MR. Common carotid artery enddiastolic velocity and acceleration time can predict degree of internal carotid artery stenosis. J Vasc Surg. (2017) 66:226-31. doi: 10.1016/j.jvs.2017.01.041

33. Yagyu T, Funabashi S, Yoneda S, Noguchi T, Yasuda S. Novel evaluation method for lower extremity peripheral artery disease with duplex ultrasound- usefulness of acceleration time. Circ J. (2020) 84:1990-8. doi: 10.1253/circj.CJ-20-0427

34. Burdick L, Airoldi F, Marana I, Giussani M, Alberti C, Cianci M, et al. Superiority of acceleration and acceleration time over pulsatility and resistance indices as screening tests for renal artery stenosis. J Hypertens. (1996) 14:1229-35. doi: 10.1097/00004872-199610000-00012

35. Dodd GD, Memel DS, Zajko AB, Baron RL, Santaguida LA. Hepatic artery stenosis and thrombosis in transplant recipients: doppler diagnosis with 
resistive index and systolic acceleration time. Radiology. (1994) 192:657-61. doi: 10.1148/radiology.192.3.8058930

36. Takemoto K, Hirata K, Wada N, Shiono Y, Komukai K, Tanimoto T, et al. Acceleration time of systolic coronary flow velocity to diagnose coronary stenosis in patients with microvascular dysfunction. J Am Soc Echocardiogr. (2014) 27:200-7. doi: 10.1016/j.echo.2013.10.013

Conflict of Interest: The authors declare that the research was conducted in the absence of any commercial or financial relationships that could be construed as a potential conflict of interest.

Publisher's Note: All claims expressed in this article are solely those of the authors and do not necessarily represent those of their affiliated organizations, or those of the publisher, the editors and the reviewers Any product that may be evaluated in this article, or claim that may be made by its manufacturer, is not guaranteed or endorsed by the publisher.

Copyright (0) 2022 Zhang, Wang, Chen, Wang, Xing and Cui. This is an open-access article distributed under the terms of the Creative Commons Attribution License (CC $B Y)$. The use, distribution or reproduction in other forums is permitted, provided the original author(s) and the copyright owner(s) are credited and that the original publication in this journal is cited, in accordance with accepted academic practice. No use, distribution or reproduction is permitted which does not comply with these terms. 